\title{
Correction to: Development and validation of EST-SSR markers of Magnolia wufengensis using de novo transcriptome sequencing
}

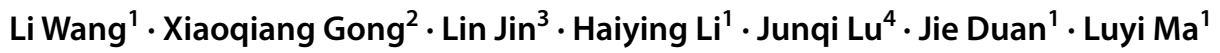

Published online: 29 May 2019

○) Springer-Verlag GmbH Germany, part of Springer Nature 2019

\section{Correction to: Trees}

https://doi.org/10.1007/s00468-019-01853-2

Unfortunately, the word "markers" was incorrectly spelled in the title of original version. The complete correct title is given below.

Development and validation of EST-SSR markers of Magnolia wufengensis using de novo transcriptome sequencing

The original article is updated.

The original article can be found online at https://doi.org/10.1007/ s00468-019-01853-2.

Jie Duan

duanjiebfu@vip.qq.com

$\triangle$ Luyi Ma

maluyi@bjfu.edu.cn

1 Key Laboratory for Silviculture and Conservation of the Ministry of Education, College of Forestry, Beijing Forestry University, Beijing 100083, China

2 College of Forestry, Beijing Forestry University, Beijing 100083, China

3 Institute of Chinese Medicinal Materials, Nanjing Agricultural University, Nanjing 210095, China

4 Department of Molecular and Cellular Biology, University of California, Davis, Davis, CA 95616, USA 